\title{
Comunicação
}

[Communication]

\section{Radiologia do intestino delgado de cães por meio da técnica de duplo-contraste}

\author{
[Small bowel double-contrast study in dogs] \\ S.F. Rausch ${ }^{1}$, C.L.B. Godoy ${ }^{1}$, C. Schmidt $^{1}$, L.C. Pellegrini ${ }^{1}$ D.C. Veiga ${ }^{2}$, G. Krolikowski ${ }^{3}$, \\ L.B. Wolle ${ }^{3}$, A.N.C. Oliveira ${ }^{3}$ \\ ${ }^{1}$ Universidade Federal de Santa Maria-UFSM \\ Av. Sete de Setembro, 759/407 - Passo Fundo \\ 99010-121 - Santa Maria, RS \\ ${ }^{2}$ Aluno de pós-graduação-UFSM - Santa Maria, RS \\ ${ }^{3}$ Aluno de graduação-UFSM - Santa Maria, RS
}

A escolha do método de diagnóstico por imagem, a ser usado nas alterações do trato gastrintestinal (TGI) está embasada na suspeita clínica dos segmentos afetados e na disponibilidade dos métodos tecnológicos a serem aplicados (Kleine e Lamb, 1989).

Em medicina veterinária, a radiologia permanece sendo o exame mais utilizado para avaliação do intestino delgado (ID). Exames radiográficos simples podem fornecer diagnósticos em situações nas quais há evidência de alterações de densidade, posição, tamanho e contorno das alças intestinais. Quando o exame radiográfico simples não é satisfatoriamente diagnóstico, um exame contrastado com meio de contraste positivo (MCP) pode ser realizado.

O MCP mais utilizado é o sulfato de bário, de uso exclusivo para o TGI. Por ter apresentações comerciais em forma de suspensões coloidais, este meio de contraste (MC) não deve ser utilizado por via intravenosa, e a dose utilizada para opacificação do TGI de cães, segundo Root (1987), varia entre 6 a $12 \mathrm{~mL} / \mathrm{kg}$, por via oral ou através de sondagem gástrica. Durante a administração por via oral (Root, 1987; Kealy e McAllister, 2000), deve-se ter cuidado para que não ocorram falsa via e aspiração, podendo resultar em pneumonia, dessa forma, Ackermann (2002) e Jung et al. (2003) indicam a sondagem gástrica.

Recebido em 1 de agosto de 2008

Aceito em 4 de fevereiro de 2009

E-mail: sfrausch@terra.com.br
Segundo Kealy e McAllister (2000), o padrão da superfície mucosa dos cães não é tão evidente como nos humanos, assim, diferentemente, estudos com sulfato de bário demonstram um padrão de superfície mucosa fimbriado no ID de cães.

Radiografias em tempos pré-estabelecidos são obtidas após a administração do MCP, a critério do radiologista (Root, 1987; Kealy e McAllister, 2000; Jung et al. 2003), sendo que o tempo total de passagem do MC pelo ID é de aproximadamente 180 minutos, ainda que uma variação de 90 minutos seja considerada normal. As projeções radiográficas utilizadas são lateral direita e esquerda, ventrodorsal, dorsoventral e obliquadas (Ackerman, 2002).

O estudo realizado por Jung et al. (2003) comparou o uso isolado do meio de contraste positivo e quando adicionado de hidroxipropilmetilcelulose como meio de contraste negativo (MCN). Esse último demonstrou melhora significativa na visualização das alças intestinais pela radiotransparência, quando comparado ao MCP que promove grande sobreposição destas. Os cães submetidos a este exame receberam $4 \mathrm{mg} / \mathrm{kg}$ de sulfato de bário, o $\mathrm{MCP}$, e $15 \mathrm{~mL} / \mathrm{kg}$ de MCN de média viscosidade, e foi obtido um tempo total de trânsito de aproximadamente 96 minutos. A sondagem do ID para infusão dos meios de contraste foi feita com fluoroscopia e, após, radiografias seriadas 
em duas projeções perpendiculares entre si foram obtidas.

Em medicina humana, o estudo radiográfico com a administração de meio de contraste positivo, continua sendo o método de eleição na demonstração de lesões da superfície mucosa, já que possui baixo custo e alta sensibilidade. Sua limitação deve-se à distensão das alças intestinais pelo MCP, que se sobrepõem, dificultando a visualização de alterações (Nacif et al., 2004). Maglinte et al. (1982) demonstraram que muitas lesões do ID podem passar despercebidas ao exame com MCP. Os autores consideram dois tipos de erros de diagnóstico, o cometido pelo radiologista, ao deixar de percebê-las e o causado pela técnica radiográfica inadequada, que dificulta ou até mesmo impede a visualização das lesões.

Em humanos, este exame radiográfico é feito na maioria das vezes sob sondagem intestinal via nasal, posicionada na junção duodeno-jejunal, onde não há influência da modulação pilórica no esvaziamento gástrico, não interferindo na quantidade de meio de contraste que atinge o intestino delgado em certo espaço de tempo. Ambos os contrastes, sulfato de bário e carboximetilcelulose (CMC), meios de contraste positivo e negativo, respectivamente, são injetados por meio de uma bomba peristáltica (Oliveira et al., 1997), ou simplesmente por gravidade (Antes e Lissner, 1983). Outro MCN passível de emprego para a promoção do duplocontraste do ID é o ar ambiente (Lorimier et al., 1956; Kobayashi, 1988).

Essa técnica em humanos, permite uma melhor visualização da superfície mucosa em relação ao estudo realizado somente com MCP e também diminui significativamente a sobreposição das alças intestinais, uma vez que estas podem ser vistas através da transparência das mesmas (Oliveira et al., 1997).

Este estudo tem por objetivos adequar e avaliar técnicas radiográficas utilizadas em radiologia humana, para utilização em medicina veterinária, promovendo melhor visualização do ID de cães por meio do duplo-contraste e determinar o melhor MCN para o emprego nesta técnica, comparando o ar ambiente e uma solução de carboximetilcelulose.

Foram utilizados para o exame do intestino delgado por meio da técnica de duplo-contraste três grupos de 10 cães, adultos, de ambos os sexos, pesando entre 4 e $17 \mathrm{~kg}$, sem raça definida e clinicamente sadios. No grupo 1, a técnica foi testada com sulfato de bário como meio de contraste positivo, e ar ambiente como negativo. Os cães do grupo 2 receberam carboximetilcelulose como MCN e os do grupo 3 , os mesmos meios de contraste do grupo 1, porém com menor dose de sulfato de bário.

As dosagens do meio de contraste positivo foram obtidas a partir do ajuste entre as utilizadas na gastrografia com duplo-contraste e a utilizada para opacificação do intestino delgado, até que se obtivesse somente uma fina camada que recobrisse a superfície mucosa. $\mathrm{O}$ ar ambiente teve sua dose modificada a partir da utilizada na gastrografia com duplo-contraste, e a carboximetilcelulose foi diminuída em relação à utilizada por Oliveira et al. (1997) em humanos. As dosagens dos meios de contraste estão demonstradas na Tab. 1.

Tabela 1. Dosagens dos meios de contraste utilizadas nos grupos de cães submetidos ao exame

\begin{tabular}{cccc}
\hline & \multicolumn{3}{c}{ Meios de contraste } \\
\cline { 2 - 4 } & $\begin{array}{c}\text { Sulfato de bário } \\
(\mathrm{mL} / \mathrm{kg})\end{array}$ & $\begin{array}{c}\text { Carboximetilcelulose } \\
(\mathrm{mL} / \mathrm{kg})\end{array}$ & $\begin{array}{c}\text { Ar ambiente } \\
(\mathrm{mL} / \mathrm{kg})\end{array}$ \\
\hline Grupo 1 & 4 & - & 20 \\
Grupo 2 & 4 & 50 & - \\
Grupo 3 & 3 & - & 20 \\
\hline
\end{tabular}

Os animais foram submetidos a jejum alimentar de aproximadamente vinte e quatro horas, antes da realização do exame, sem qualquer restrição à ingestão de água. Rotineiramente, antes do exame contrastado, radiografias simples em duas projeções perpendiculares entre si, lateral direita e ventrodorsal, foram obtidas. Após a certificação de ausência de conteúdo no trato gastrintestinal ou evidência radiográfica de alteração abdominal, foi administrada 
metoclopramida $^{1}$ na dosagem de $1,5 \mathrm{mg} / \mathrm{kg}$ via intravenosa lenta, na intenção de promover relaxamento pilórico, diminuindo sua modulação na passagem dos meios de contraste.

Nos animais do grupo 1, passados cinco minutos da administração da metoclopramida, foi administrado sulfato de bário $^{2}$ a $100 \%$ em dose de $4 \mathrm{~mL} / \mathrm{kg}$, por via oral. Após 15 minutos, foram feitas rotações de $360^{\circ}$ do paciente, para que houvesse a cobertura total da mucosa duodenal pelo sulfato de bário. Logo a seguir, o MCN (ar ambiente) foi administrado via sonda oroesofágica em uma dosagem de $20 \mathrm{~mL} / \mathrm{kg}$. Aos animais do grupo 2, após a administração do MCP feita da mesma forma que no primeiro, o meio de contraste negativo empregado foi a $\mathrm{CMC}^{3}$ também administrado via sonda oroesofágica, na dose de $50 \mathrm{~mL} / \mathrm{kg}$. Nos animais do grupo 3, administrou-se os mesmos meios de contraste utilizados no primeiro, empregados da mesma forma, porém a dose do sulfato de bário foi reduzida a $3 \mathrm{~mL} / \mathrm{kg}$ (Fig. 1).

Os três grupos de animais foram submetidos a sequiências radiográficas semelhantes. A primeira foi realizada em duas projeções perpendiculares entre si, uma lateral e uma ventrodorsal, imediatamente após a administração do meio de contraste negativo. A segunda seqüência, 20 minutos após o início da primeira administração do meio de contraste negativo, submetendo o paciente à nova administração deste MC, efetuando-se as tomadas radiográficas. Repetiuse então a metodologia, passados outros 20 minutos, completando um total de três seqüências, que foram suficientes para a visualização de toda a extensão do ID, por meio da técnica de duplo-contraste.

Embora, a cada sequiência, as radiografias tenham sido avaliadas em relação ao posicionamento e ao deslocamento do MC, após o término de cada exame, houve o estudo minucioso dos resultados obtidos. Foram adotados como critérios de avaliação do exame o

\footnotetext{
${ }^{1}$ Plasil - Hoeschst Marion Roussel - Av. Das Nações Unidas, $18001-8^{\circ}$ andar - São Paulo - SP

${ }^{2}$ Bariogel - Cristália - Rod. Itapira-Lindóia, km 14 - Itapira - SP

${ }^{3}$ Carboximetilcelulose 2,5g - Dermapelle Farmácia de Manipulação - Rua do Acampamento, 192 - Santa Maria RS
}

padrão das radiografias em relação aos fatores de exposição e o posicionamento adequado do animal na radiografia. Também, a cobertura da superfície mucosa promovida por uma fina camada de meio de contraste positivo, e a distensão e transparência das alças do intestino delgado pelo meio de contraste negativo.

De acordo com Nacif et al. (2004), o estudo radiográfico do intestino delgado com meio de contraste positivo, em humanos, demonstra alças intestinais distendidas e repletas, dificultando a demonstração das lesões pela sobreposição. Conforme Oliveira et al. (1997) e Jung et al. (2003), quando há duplo-contraste no interior das alças intestinais, existe uma melhor visualização da superfície mucosa e menor sobreposição, pois as alças são vistas pela radiotransparência. Os resultados obtidos assemelham-se aos de autores citados, pois por meio do duplo-contraste intestinal houve a adequada visualização pela radiotransparência.

Radiograficamente, no presente estudo não foram observadas diferenças significativas em relação ao meio de contraste negativo empregado, porém a CMC, além de necessitar de preparo prévio de 24 horas, é uma técnica com maior custo. Dessa forma, o ar ambiente demonstra ser de eleição para o estudo.

O MCN foi administrado via sondagem oroesofágica, principalmente por sua grande quantidade e falta de palatabilidade, no caso da CMC, mas nenhum dos pacientes demonstrou reação significativa de desconforto mediante a passagem da sonda. Ackerman (2002) e Jung et al. (2003) indicaram a sondagem para a administração dos MC, pois dessa forma ocorreria menor perda, evitar-se-ia a inalação e não ocorreria demasiada aerofagia, ao contrário da administração por via oral. Já o MCP foi administrado por via oral, segundo a indicação de Root (1987) e Kealy e McAllister (2000), e não foram observados sinais de desconforto ou de aspiração do MCP, e as perdas foram mínimas. 

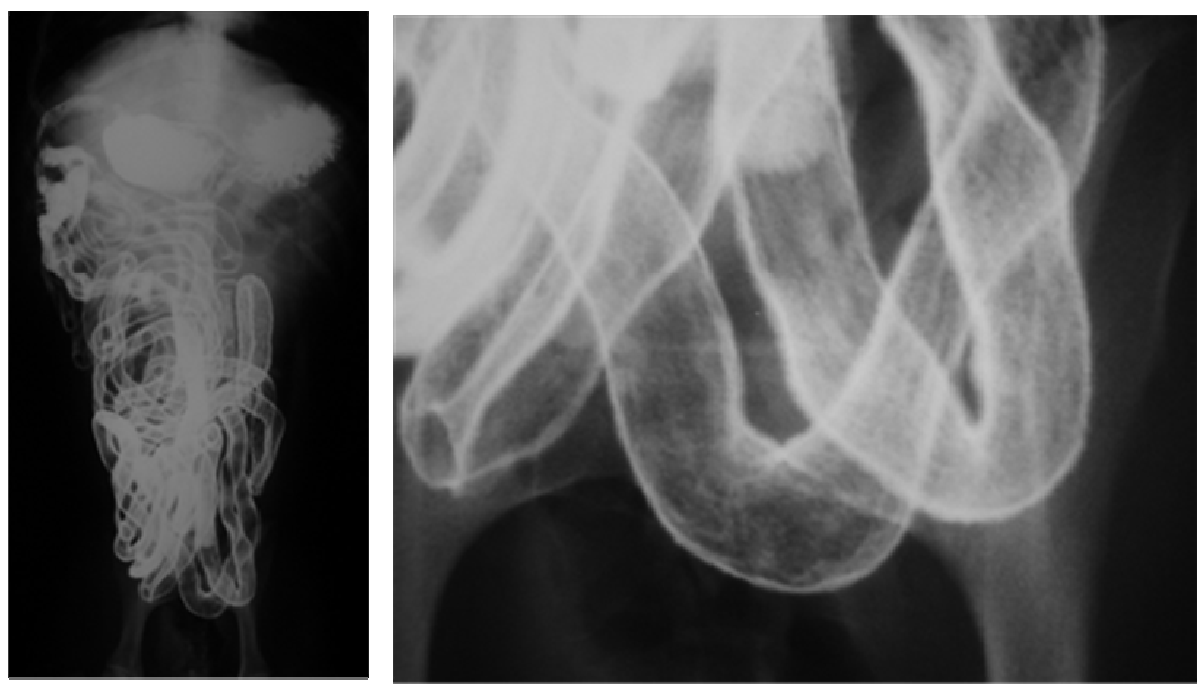

Figura 1. Radiografias abdominal em projeção ventrodorsal (A) e detalhe dela (B), após a administração do sulfato de bário (meio de contraste positivo) e ar ambiente (meio de contraste negativo), demonstrando o duplo contraste do intestino delgado.

De acordo com Kealy e McAllister (2000), os tempos para a realização das radiografias são pré-estabelecidos e a primeira tomada deve ser realizada imediatamente, após a administração do meio de contraste. A segunda, a cada 20 minutos, na primeira hora e, então, a cada hora, conforme indicação do radiologista. Root (1987) indicou que a segunda radiografia seja feita após cinco minutos, então aos 15, 30 e 60 minutos, e a cada meia hora, até que o MC esteja no intestino grosso. Ainda, de acordo com Jung et al. (2003), após a saída do meio de contraste positivo do estômago, radiografias seqüenciais devem ser feitas a cada 10 minutos. Neste estudo, os tempos também foram pré-estabelecidos, porém não seguiram os indicados pela literatura, pois a metoclopramida aumenta a motilidade do ID, promovendo uma rápida passagem dos meios de contraste até o intestino grosso.

A administração deste fármaco foi feita de acordo com Oliveira et al. (1997), ao contrário de Jung et al. (2003), que não a empregaram em cães, atingindo um tempo de trânsito no ID de aproximadamente 96 minutos. Neste estudo, a média foi de 35 minutos, porém não foi passível de comparação, pois houve aumento da motilidade pelo emprego da metoclopramida, diminuindo o tempo total de trânsito.

Dos 10 cães estudados no grupo 3, somente um não teve a passagem dos meios de contraste dentro do tempo esperado, chegando ao ID em 30 minutos ao invés de 15 , possivelmente devido a uma resposta individual do animal, pois ocorreu apenas uma vez em 10 exames utilizando o mesmo protocolo. Nos 30 cães, não houve efeitos colaterais como vômitos e diarréia, da mesma forma como observaram Jung et al. (2003).

Os resultados obtidos permitem concluir que a técnica de duplo-contraste proporciona imagem ideal da superfície mucosa do intestino delgado, e é eficaz com o emprego de sulfato de bário, administrado por via oral, e ar ambiente, administrado por sondagem gástrica, sob relaxamento pilórico promovido pela metoclopramida. $\mathrm{O}$ ar ambiente foi eleito como melhor meio de contraste negativo com menor custo. O tempo em que os meios de contraste atingem o final do intestino delgado é, em média, 35 minutos, devido a utilização da metoclopramida, fazendo com que este exame seja rápido. $\mathrm{O}$ exame é de fácil execução, com mínima exposição de pessoal e pacientes à radiação ionizante, baixo custo e eficácia na exploração anatômica, podendo, desta forma, ser incorporado à rotina dos setores de radiodiagnóstico veterinário.

Palavras-chave: cão, intestino delgado, radiologia, duplo contraste 


\begin{abstract}
Radiographic exams still the main form to evaluate small bowel in all its extension, and when survey projections are not diagnostic, barium series are used although its sensibility can be imitated by filled overlapped loops, difficulting detailed mucosa visualization. The objective of this study was to adjust an exam technique with intestinal double-contrast, based on techniques used in the human medicine, which resulted satisfactorily in the demonstration of the mucosal surface in the 30 dogs submitted to the exam. The double-contrast in the lumen was achieved by a combination of a positive contrast medium, recovering the mucosal surface, and a negative filling the lumen. Ambient air and carboximethylcelullose were the negative contrast medium tested and both were radiographicaly similar.
\end{abstract}

Keywords: dog, small bowel, radiology, double-contrast

\section{REFERÊNCIAS BIBLIOGRÁFICAS}

ACKERMAN, N. Imaging the GI tract. In: WSAVA proceedings. Davis: Veterinary information network, 2002. Disponível em <http://www.vin.com/proceedings/Proceedings.p lx?CID=WSAVA2002\&PID=2604>. Acessado em 15 jan. 2005.

ANTES, G.; LISSNER, J. Double-contrast small-bowel examination with barium and methylcellulose. Radiology, v.148, p.37-40, 1983.

JUNG, J.; CHOI, M.; CHANG, J. et al. Effect of methylcelullose on upper gastrointestinal quality in dogs. Vet Radiol Ultrasound, v.44, p.642-645, 2003.

KEALY, J.K.; McALLISTER, H. The abdomen. In:__. Diagnostic radiology and ultrasonography of the dog and cat. 3.ed. Philadelphia: W.B. Saunders, 2000. p.60-95.

KLEINE, L.J.; LAMB, C.R. Comparative organ imaging: the gastrointestinal tract. Vet. Radiol., v.30, p.133-141, 1989.

KOBAYASHI, S. A method for double contrast radiography of the small intestine. J Belge Radiol, v.71, p.365-374, 1988.
LORIMIER, A.A.; MOEHRING, H.G.; HANNAN, J.R. Clinical roentgenology. Springfield: Charles C. Thomas, 1956. v.4.

MAGLINTE, D.D.; BURNEY, B.T.; MILLER, R.E. Lesions missed on small-bowel followthrough: analysis and recommendations. Radiology, v.144, p.737-739, 1982.

NACIF, M.S.; ROCHA, V.M.; MELLO, R.A. et al. Análise retrospectiva do trânsito do delgado em um serviço de radiologia de hospital geral. Radiol. Bras., v.37, p.179-183, 2004.

OLIVEIRA, J.M.; COSTACURTA, M.A.; GANC, A.J. O valor da sedação e da bomba peristáltica elétrica para a infusão dos meios de contraste no tempo de realização da enteróclise bifásica com carboximetilcellose a $0,5 \%$ : análise de 55 casos. Radiol. Bras., v.30, p.189-203, 1997.

ROOT, C.R. Radiografia contrastada do trato alimentar. In: TICER, J.W. Técnicas radiográficas na prática veterinária. São Paulo: Roca, 1987. p.352-380. 